\title{
Balloon-expandable transaortic transcatheter aortic valve implantation with or without predilation
}

\author{
Nikolaos Bonaros, MD, PhD, ${ }^{a}$ Markus Kofler, MD, ${ }^{a}$ Derk Frank, MD, ${ }^{b}$ Riccardo Cocchieri, MD, \\ Dariusz Jagielak, MD, ${ }^{\mathrm{d}}$ Marco Aiello, MD, ${ }^{\mathrm{e}}$ Joel Lapeze, MD,${ }_{\mathrm{f}}^{\mathrm{f}}$ Mika Laine, MD, ${ }^{\mathrm{g}}$ Sidney Chocron, MD, ${ }^{\mathrm{h}}$ \\ Douglas Muir, MD, ${ }^{\mathrm{i}}$ Walter Eichinger, MD, ${ }^{\mathrm{j}}$ Matthias Thielmann, MD,${ }^{\mathrm{k}}$ Louis Labrousse, MD, ${ }^{1}$ \\ Vinayak Bapat, MD, ${ }^{\mathrm{m}}$ Kjell Arne Rein, MD, ${ }^{\mathrm{n}}$ Jean-Philippe Verhoye, MD, ${ }^{\mathrm{o}}$ Gino Gerosa, MD, ${ }^{\mathrm{p}}$ \\ Hardy Baumbach, MD, ${ }^{\mathrm{q}}$ Cornelia Deutsch, MD,${ }^{\mathrm{r}}$ Peter Bramlage, MD, ${ }^{\mathrm{r}}$ Martin Thoenes, MD, ${ }^{\mathrm{s}}$ and \\ Mauro Romano, $\mathrm{MD}^{\mathrm{t}}$
}

\section{ABSTRACT}

Objective: It has been reported that balloon aortic valvuloplasty immediately before transfemoral or transapical transcatheter aortic valve implantation has mostly little to no clinical value. We aimed to provide data on the need for balloon aortic valvuloplasty in patients undergoing transaortic transcatheter aortic valve implantation.

Methods: Patients undergoing transaortic transcatheter aortic valve implantation with the Edwards SAPIEN XT (Nyon, Switzerland) or 3 transcatheter heart valve were prospectively included at 18 sites across Europe. In the present analysis, we compare the periprocedural and 30-day outcomes of patients undergoing conventional $(+$ balloon aortic valvuloplasty) versus direct ( - balloon aortic valvuloplasty) transaortic transcatheter aortic valve implantation.

Results: Of the 300 patients enrolled, 222 underwent conventional and 78 underwent direct transaortic transcatheter aortic valve implantation. Peak and mean transvalvular gradients were improved in both groups with no significant difference between groups. Procedural duration, contrast agent volume, and requirement for postdilation were also comparable. A trend toward fewer periprocedural complications was evident in the direct group (3.9\% vs $11.3 \%$; $P=.053)$, with significantly lower rates of permanent pacemaker implantation $(0 \%$ vs $5.0 \% ; P=.034)$. Balloon aortic valvuloplasty omission had no significant effect on any of the 30-day safety and efficacy outcomes, including Valve Academic Research Consortium-2 composite end points (early safety events: $22.7 \%$ vs $17.4 \%$, odds ratio, $1.17,95 \%$ confidence interval, $0.53-2.62$; clinical efficacy events: $20.5 \%$ vs $18.7 \%$, odds ratio, $1.14,95 \%$ confidence interval, $0.51-2.55)$.

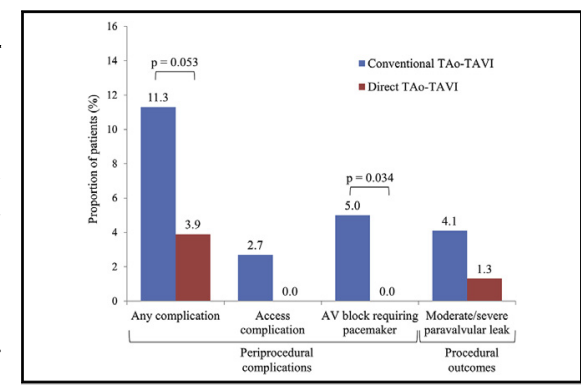

Periprocedural complications and procedural outcomes in conventional versus direct TAo-TAVI.

\section{Central Message}

Predilation in transaortic TAVI procedures using an Edwards SAPIEN (Nyon, Switzerland) balloonexpandable valve seems to have little clinical value for many patients and may result in a higher rate of PPI.

\section{Perspective}

Although BAV predilation in TA- and TF-TAVI seems to be of little clinical value, evidence for this in TAo-TAVI is lacking. Our real-world data reveal that many findings from TA and TF studies also apply to TAo-TAVI and suggest that omission of predilation may reduce periprocedural PPI. This is valuable knowledge for informing surgical decisions and adds to the limited data on TAo-TAVI.

See Editorial Commentary page 924.

\footnotetext{
From the ${ }^{\mathrm{a} D e p a r t m e n t}$ of Cardiac Surgery, Medical University Innsbruck, Innsbruck, Austria; ${ }^{b}$ Department of Internal Medicine III (Cardiology and Angiology) UKSH, Campus Kiel, Kiel, Germany; ${ }^{c}$ Heart Centre, Academic Medical Centre, University of Amsterdam, Amsterdam, The Netherlands; ${ }^{\mathrm{d}}$ Department of Cardiac and Vascular Surgery, Medical University of Gdansk, Gdansk, Poland; ${ }^{\mathrm{e}}$ Department of Cardiothoracic Surgery, Foundation IRCCS Policlinico S Matteo, Pavia University School of Medicine, Pavia, Italy; ${ }^{\mathrm{f}}$ Department of Cardiovascular Surgery, Hospital Louis Pradel, Lyon, France; ${ }^{\mathrm{g}}$ Division of Cardiology, Helsinki University Central Hospital, Helsinki, Finland; ${ }^{h}$ Cardiothoracic Surgery, Hospital Jean Minjoz, University Hospital of Besancon, Besancon, France; ${ }^{\mathrm{i} C a r d i o t h o r a c i c ~ D i v i s i o n, ~}$ James Cook Hospital, Middlesbrough, United Kingdom; ${ }^{j}$ Department of Cardiothoracic Surgery, Klinikum Bogenhausen, Munich, Germany; ${ }^{k}$ Department of Thoracic and Cardiovascular Surgery, West-German Heart Centre, University Hospital Essen, Essen, Germany; ${ }^{1}$ Department of Cardiovascular Surgery, CHU Hospital of Bordeaux, Bordeaux, France; ${ }^{\mathrm{m}} \mathrm{St}$ Thomas' Hospital, London, United Kingdom; ${ }^{\text {n} D e p a r t m e n t ~ o f ~ C a r d i o t h o r a c i c ~ S u r g e r y, ~ R i k s h o s p i t a l ~ O s l o, ~ O s l o, ~ N o r-~}$ way; ${ }^{\circ}$ Department of Cardiovascular Surgery, CHU Rennes, Rennes, France; ${ }^{\mathrm{P} D e-}$
}

partment of Cardiac Surgery, University of Padova, Padova, Italy; ${ }^{\mathrm{q}}$ Department of Cardiovascular Surgery, Robert-Bosch-Krankenhaus Stuttgart, Stuttgart, Germany; ${ }^{\mathrm{r}}$ Institute for Pharmacology and Preventive Medicine, Cloppenburg, Germany; ${ }^{\mathrm{s}}$ Edwards Lifesciences, Medical Affairs/Professional Education, Nyon, Switzerland; and ${ }^{\mathrm{t}}$ Institut Hospitalier Jacques Cartier, Massy, France.

A research grant was provided by Edwards Lifesciences (Nyon, Switzerland) to the Sponsor (IPPMed, Cloppenburg, Germany). Clinicaltrials.gov: NCT01991431. Drs Bonaros and Kofler contributed equally to the article.

Received for publication Jan 25, 2017; revisions received Sept 8, 2017; accepted for publication Oct 2, 2017; available ahead of print Dec 6, 2017.

Address for reprints: Nikolaos Bonaros, MD, PhD, Department of Cardiac Surgery, Medical University Innsbruck, Anichstrasse 35, A-6020, Innsbruck, Austria (E-mail: nikolaos.bonaros@i-med.ac.at).

0022-5223/\$36.00

Copyright (C) 2017 by The American Association for Thoracic Surgery

https://doi.org/10.1016/j.jtcvs.2017.10.071 


$$
\begin{aligned}
& \text { Abbreviations and Acronyms } \\
& \mathrm{AV} \quad=\text { atrioventricular } \\
& \mathrm{BAV}=\text { balloon aortic valvuloplasty } \\
& \text { CI = confidence interval } \\
& \text { NYHA }=\text { New York Heart Association } \\
& \text { OR = odds ratio } \\
& \text { PAD }=\text { peripheral artery disease } \\
& \text { PPI = permanent pacemaker implantation } \\
& \text { PVL = paravalvular leak } \\
& \text { ROUTE }=\text { Registry Of the Utilization of the TAo- } \\
& \text { TAVI approach using the Edwards } \\
& \text { SAPIEN Valve } \\
& \text { STS = Society of Thoracic Surgeons } \\
& \mathrm{TA}=\text { transapical } \\
& \text { TAo }=\text { transaortic } \\
& \text { TAVI }=\text { transcatheter aortic valve implantation } \\
& \text { TF }=\text { transfemoral } \\
& \text { THV }=\text { transcatheter heart valve } \\
& \text { VARC-2 = Valve Academic Research Consortium-2 }
\end{aligned}
$$

Scanning this $\mathrm{QR}$ code will take you to a supplemental video for the article.

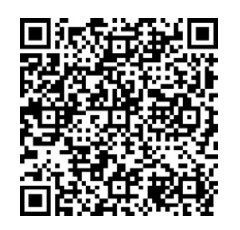

Conclusions: For many patients, balloon aortic valvuloplasty predilation seems to have little clinical value in transaortic transcatheter aortic valve implantation using a balloon expandable transcatheter valve and may result in a higher rate of periprocedural complications, particularly in terms of permanent pacemaker implantation. ( $\mathrm{J}$ Thorac Cardiovasc Surg 2018;155:915-23)

Balloon aortic valvuloplasty (BAV) predilation has generally been considered fundamental to the transcatheter aortic valve implantation (TAVI) procedure to allow prior assessment of the appropriate transcatheter heart valve (THV) size, facilitate the crossing of the aortic annulus, and maximize the expansion of the prosthesis to minimize paravalvular leak (PVL). ${ }^{1}$ However, predilation may contribute to stroke and embolic event rates because of a marked

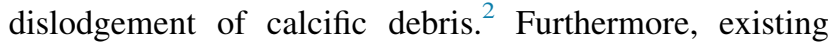
studies on transfemoral (TF) and transapical (TA) TAVI have all reported that omitting the predilation step (known as "direct TAVI") results in a level of efficacy similar to that of the conventional approach (+BAV predilation), with potential additional safety advantages. ${ }^{3-5}$ The latter include lower rates of permanent pacemaker implantation (PPI) and 30-day mortality, ${ }^{6}$ shorter procedural and fluoroscopy times, ${ }^{3}$ and reduced contrast agent volumes. ${ }^{3,7}$ In addition, several studies suggest a lower degree of PVL after direct versus conventional TAVI, ${ }^{3,8}$ potentially discrediting the aforementioned belief that BAV predilation is imperative for successful THV landing and apposition. Despite the accumulating data for TF- and TA-TAVI, no studies comparing direct with conventional TAo-TAVI have been published. Patients undergoing TAo-TAVI are of particular interest, not only as a distinct population but also because of key procedural characteristics. Although TAVI via the short, antegrade TA access route can be easily performed without BAV, its omission during retrograde TF-TAVI is complicated by the long distance between the femoral access point and the aortic valve, and by the unequal distribution of forces applied by the surgeon at the groin to different points of the catheter. This is a particularly relevant problem in patients with a horizontal annulus, severe kinking of the iliac vessels, or very sharp angulation of the aortic arch. TAo-TAVI is the only approach that combines retrograde insertion with direct transmission of forces to the stenotic aortic valve because of the proximity and angulation of the catheter entry site. As such, TAo-TAVI theoretically can be used in the majority of patients. The value of BAV predilation under these particular circumstances merits evaluation.

The present analysis of the Registry Of the Utilization of the TAo-TAVI approach using the Edwards SAPIEN Valve (ROUTE) (Nyon, Switzerland) specifically focuses on the clinical value of BAV predilation in TAo-TAVI. Our aim was to evaluate the impact of the direct approach on procedural aspects and short-term event rates in patients with severe AS undergoing this procedure with the Edwards SAPIEN XT or 3 THV.

\section{MATERIALS AND METHODS \\ Design}

Data from the multicenter, multinational, prospective ROUTE registry (NCT01991431) were used in the present analysis, the design and rationale of which have been described. ${ }^{9,10}$ This registry was established in February 2013, with the aim of determining the overall safety and efficacy of the TAo-TAVI approach. It received prior institutional review board approval from the relevant ethics committees at each site. All patients included in the registry provided written informed consent.

\section{Study Participants}

Patients were consecutively enrolled at 18 sites across Europe. Criteria for inclusion were (1) age 18 years or more; (2) severe AS (defined as aortic valve surface area $<1.0 \mathrm{~cm}^{2}$ plus a mean transvalvular gradient $>40 \mathrm{~mm}$ $\mathrm{Hg}$ ); (3) scheduled to undergo TAo-TAVI using the Edwards SAPIEN $\mathrm{XT}$ or $3 \mathrm{THV}$; and (4) complying with the indications for this procedure detailed in the "Instructions for Use." Patients scheduled to undergo a concomitant procedure were excluded. For more details on inclusion and exclusion criteria, readers are referred to the registry design article. ${ }^{9}$ 


\section{Transaortic-Transcatheter Aortic Valve Implantation Procedure}

In the present analysis, all patients who underwent the procedure with the BAV predilation step (Video 1) included were classified as the "conventional TAo-TAVI" group, whereas all those who underwent the procedure with the BAV predilation step omitted were classified as the "direct TAoTAVI" group. The decision as to whether or not to perform BAV predilation was made at the discretion of the treating physician, independent of inclusion in the registry.

\section{Objectives}

The primary objective of the present analysis was to compare the proportion of patients in the direct versus conventional TAo-TAVI group meeting Valve Academic Research Consortium-2 (VARC-2) early safety and efficacy composite end points at 30 days. ${ }^{11}$ Early safety was defined as a composite of all-cause mortality, nonfatal stroke/transient ischemic attack, life-threatening bleeding, acute kidney injury stage 2 or 3, major vascular complication, and valve-related dysfunction. Early efficacy was defined as a composite of all-cause mortality, stroke, hospitalization for valve-related symptoms, New York Heart Association (NYHA) class III or IV, and valve-related dysfunction. The secondary objectives were to establish the effect of BAV predilation on individual 30-day complication/event rates, as well as on periprocedural outcomes such as complication rates, procedural time, need for balloon postdilation, and contrast agent use.

\section{Statistical Analysis}

All of the collected data were entered into a database by a physician or study nurse via electronic case report forms; more than $20 \%$ of patients were subjected to source data verification after registry completion.

All relevant data are expressed using descriptive statistics. Categoric variables are presented as absolute numbers and percentages, and continuous variables are given as means \pm standard deviation or median (interquartile range). For comparisons between the conventional and direct group, a chi-square test or Fisher exact test was used for categoric variables, a $t$ test for normally distributed continuous variables, and a Mann-Whitney $U$ test for non-normally distributed continuous variables. $P$ values are of descriptive nature only and are adjusted for multiplicity. A logistic

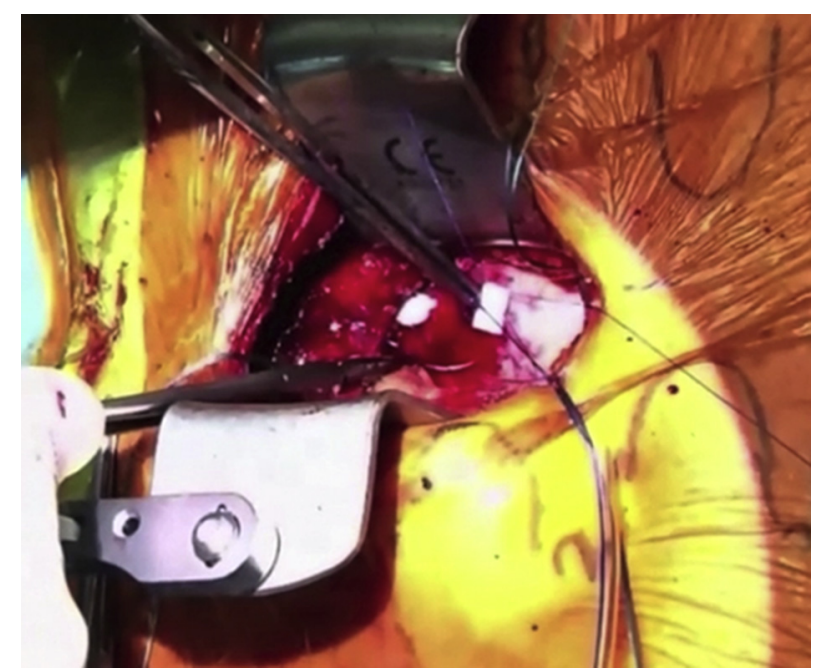

VIDEO 1. Video showing the TAo-TAVI surgical procedure. Video available at: http://www.jtcvsonline.org/article/S0022-5223(17)32400-5/fulltext.

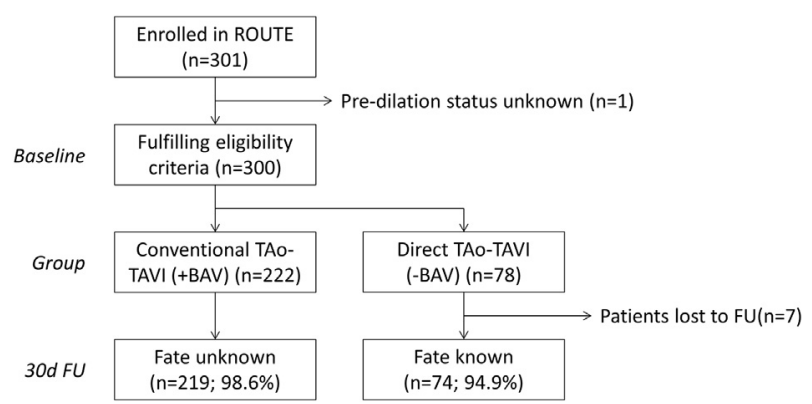

FIGURE 1. Patient flow for present analysis. ROUTE, Registry Of the Utilization of the TAo-TAVI approach using the Edwards SAPIEN Valve; TAo-TAVI, transaortic transcatheter aortic valve implantation; $B A V$, balloon aortic valvuloplasty; $F U$, follow-up.

regression adjusting for baseline characteristics (age, peripheral artery disease [PAD], moderate/severe regurgitation insufficiency, and NYHA class III or IV and prior BAV) was used to evaluate the effect of omitting BAV predilation on 30-day safety and efficacy outcomes. These data are presented as odds ratios (ORs) with $97.5 \%$ confidence intervals (CIs). A multiplicity adjusted alpha level of 0.025 was used. All data analysis was carried out using Statistical Analysis System version 9.3 (SAS Institute Inc, Cary, NC).

\section{RESULTS}

A total of 301 patients who underwent TAo-TAVI were enrolled in the ROUTE registry (Figure 1). Of these, 300 patients were eligible for the present analysis. Accordingly, study participants were grouped into 222 patients $(74 \%)$ who underwent conventional TAo-TAVI (+BAV predilation) and 78 patients $(26 \%)$ who underwent direct TAoTAVI (-BAV predilation)

\section{Baseline Patient Details}

Overall, patients had a mean age of 81.7 years and a body mass index of $26.4 \mathrm{~kg} / \mathrm{m}^{2}$, and $53.7 \%$ were female, with no significant differences between the conventional and direct groups (Table 1). The proportions of patients with prior cardiovascular interventions were also statistically comparable (35.7\% overall); however, a significantly higher proportion of the direct group had received prior BAV $(9.0 \%$ vs $2.7 \%$ of the conventional group; $P=.019)$. Surgical risk determined by the Society of Thoracic Surgeons (STS) algorithm was comparable between groups ( $9 \%$ overall). Of the comorbidities documented, only PAD was found to differ significantly between groups, affecting $47.8 \%$ of conventional compared with $26.9 \%$ of direct TAo-TAVI patients $(P=.001)$.

A greater proportion of the conventional group was classified as NYHA class III/IV ( $81.4 \%$ vs $60.3 \%$ of the direct group; $P=.001)$. The proportion of patients in the direct TAo-TAVI group with moderate or severe regurgitation insufficiency $(23.1 \%)$ was more than double that of the conventional TAo-TAVI group $(10.8 \% ; P=.007)$. 
TABLE 1. Baseline patient and disease characteristics

\begin{tabular}{|c|c|c|c|c|}
\hline & $\begin{array}{c}\text { Total }(\mathbf{N}=300) \\
\text { mean } \pm \text { SD/n/N }(\%)\end{array}$ & $\begin{array}{c}\text { Conventional } \\
\text { TAo-TAVI }(\mathbf{N}=\mathbf{2 2 2}) \\
\text { mean } \pm \text { SD } / \mathbf{n} / \mathbf{N}(\%)\end{array}$ & $\begin{array}{c}\text { Direct TAo-TAVI } \\
(\mathbf{N}=78) \\
\text { mean } \pm \text { SD/n/N }(\%)\end{array}$ & $\begin{array}{c}P \text { value } \\
\text { (conventional vs } \\
\text { direct TAo-TAVI) }\end{array}$ \\
\hline Age [y] & $81.7 \pm 5.9$ & $82.1 \pm 5.8$ & $80.7 \pm 6.3$ & .069 \\
\hline Female gender & $161 / 300(53.7)$ & $121 / 222(54.5)$ & $40 / 78(51.3)$ & .623 \\
\hline BMI $\left[\mathrm{kg} / \mathrm{m}^{2}\right]$ & $26.4 \pm 5.8$ & $26.3 \pm 5.6$ & $26.8 \pm 6.4$ & .537 \\
\hline Current smoker & $29 / 280(10.4)$ & $19 / 212(9.0)$ & $10 / 68(14.7)$ & .176 \\
\hline $\begin{array}{l}\text { Prior cardiovascular intervention } \\
\text { Prior BAV }\end{array}$ & $\begin{array}{c}107 / 300(35.7) \\
13 / 300(4.3)\end{array}$ & $\begin{array}{l}76 / 222(34.2) \\
6 / 222(2.7)\end{array}$ & $\begin{array}{l}31 / 78(39.7) \\
7 / 78(9.0)\end{array}$ & $\begin{array}{l}.382 \\
.019\end{array}$ \\
\hline \multicolumn{5}{|l|}{ Comorbidities } \\
\hline CAD & $183 / 300(61.0)$ & $137 / 222(61.7)$ & $46 / 78(59.0)$ & .670 \\
\hline Hypertension* & $230 / 298(76.7)$ & $174 / 220(79.1)$ & $56 / 78(71.8)$ & .187 \\
\hline Diabetes $\dagger$ & $79 / 298(26.5)$ & $55 / 220(25.0)$ & $24 / 78(30.8)$ & .321 \\
\hline Prior stroke/TIA & $43 / 300(14.3)$ & $29 / 220(13.1)$ & $14 / 78(18.0)$ & .289 \\
\hline PAD & $127 / 300(42.3)$ & $106 / 222(47.8)$ & 21/78 (26.9) & .001 \\
\hline Cancer & $59 / 300(19.7)$ & $42 / 222(18.9)$ & $17 / 78(21.8)$ & .583 \\
\hline Pulmonary disease $\ddagger$ & $78 / 300(26.0)$ & $60 / 222(27.0)$ & $18 / 78(23.1)$ & .494 \\
\hline Pulmonary hypertension $\S$ & $95 / 296(32.1)$ & $68 / 218(31.2)$ & 27/78 (34.6) & .578 \\
\hline Renal insufficiency $\|$ & $94 / 300(31.3)$ & $65 / 222(29.3)$ & 29/78 (37.2) & .196 \\
\hline Prior MI & 46/298 (15.4) & $33 / 221(14.9)$ & 13/77 (16.9) & .683 \\
\hline \multicolumn{5}{|l|}{ Symptoms } \\
\hline Dizziness with exertion & $105 / 300(35.0)$ & $78 / 222(35.1)$ & $27 / 78(34.6)$ & .934 \\
\hline CCS angina class III/IV & $40 / 280(14.3)$ & $33 / 212(15.6)$ & 7/68 (10.3) & .280 \\
\hline \multicolumn{5}{|l|}{ ECG } \\
\hline AV block & $39 / 291(13.4)$ & $29 / 215(13.5)$ & $5 / 76(6.6)$ & .324 \\
\hline LBBB & $43 / 296(14.5)$ & $33 / 220(15.0)$ & $10 / 76(13.2)$ & .593 \\
\hline RBBB & $33 / 287(11.5)$ & 23/211 (10.9) & $10 / 76(13.2)$ & .844 \\
\hline \multicolumn{5}{|l|}{ Echocardiogram } \\
\hline LVEF [\%] & $52.5 \pm 12.3$ & $52.7 \pm 11.7$ & $52.0 \pm 14.0$ & .657 \\
\hline Peak transvalvular gradient [mm $\mathrm{Hg}]$ & $71.5 \pm 23.3$ & $71.0 \pm 22.9$ & $73.1 \pm 24.5$ & .581 \\
\hline Mean transvalvular gradient $[\mathrm{mm} \mathrm{Hg}]$ & $44.3 \pm 15.3$ & $44.3 \pm 15.1$ & $44.3 \pm 16.1$ & .987 \\
\hline Moderate/severe regurgitation insufficiency & $42 / 300(14.0)$ & $24 / 222(10.8)$ & $18 / 78(23.1)$ & .007 \\
\hline NYHA class III/IV & $226 / 298(75.8)$ & $179 / 220(81.4)$ & $47 / 78(60.3)$ & .001 \\
\hline STS $[\%]$ & $9.0 \pm 7.6$ & $9.3 \pm 7.7$ & $8.3 \pm 7.2$ & .330 \\
\hline
\end{tabular}

$S D$, Standard deviation; TAo-TAVI, transaortic transcatheter aortic valve implantation; $B M I$, body mass index; $B A V$, balloon aortic valvuloplasty; $C A D$, coronary artery disease; $T I A$, transient ischemic attack; $P A D$, peripheral artery disease; $M I$, myocardial infarction; $C C S$, Canadian Cardiovascular Society; $E C G$, electrocardiogram; $A V$, atrioventricular; $L B B B$, left bundle branch block; $R B B B$, right bundle branch block; $L V E F$, left ventricular ejection fraction; NYHA, New York Heart Association; STS, Society of Thoracic Surgeons. *Defined as systolic blood pressure greater than $140 \mathrm{~mm} \mathrm{Hg}$, diastolic blood pressure greater than $90 \mathrm{~mm} \mathrm{Hg}$, or a documented history of hypertension. $\dagger$ Defined as documented history of diabetes. $\ddagger$ Defined as any condition causing or indicating impaired lung function. $\S$ Defined as mean pulmonary artery pressure greater than $25 \mathrm{~mm} \mathrm{Hg}$. $\|$ Defined as creatinine level greater than $3.0 \mathrm{mg} / \mathrm{dL}$.

\section{Periprocedural Details}

The most commonly used THV size was $26 \mathrm{~mm}$ (48.3\% overall) (Table 2). Device success was achieved in $98.7 \%$ of all patients. Procedural duration, volume of contrast agent used, and requirement for balloon postdilation were not significantly different between conventional and direct TAo-TAVI groups. Likewise, the median length of stay in the intensive care unit and time to discharge were comparable. There was no case of an inability to cross the aortic valve in the direct group, whereas this was a problem for 2 patients in the conventional group.
A borderline significant trend toward fewer procedural complications in the direct TAo-TAVI group compared with the conventional group was apparent $(3.9 \%$ vs $11.3 \% ; P=.053)$. The most common of these was complete atrioventricular (AV) block $(5.0 \%$ overall), with no statistical difference between groups. However, 2 cases of AV block occurred during the BAV predilation step in the conventional group. A significantly higher proportion of conventional TAo-TAVI patients required PPI $(5.0 \%$; $84.6 \%$ of $\mathrm{AV}$ block cases) compared with patients in the direct group $(0.0 \% ; P=.034)$. The frequencies of access complications and conversion to open surgery were not 
TABLE 2. Periprocedural and hospitalization details

\begin{tabular}{|c|c|c|c|c|}
\hline & $\begin{array}{c}\text { Total }(\mathbf{N}=300) \\
\text { mean } \pm \text { SD/n/N }(\%)\end{array}$ & $\begin{array}{c}\text { Conventional } \\
\text { TAo-TAVI }(\mathrm{N}=\mathbf{2 2 2}) \\
\text { mean } \pm \mathrm{SD} / \mathrm{n} / \mathrm{N}(\%)\end{array}$ & $\begin{array}{c}\text { Direct TAo-TAVI } \\
(\mathbf{N}=\mathbf{7 8}) \\
\text { mean } \pm \mathrm{SD} / \mathbf{n} / \mathbf{N}(\%)\end{array}$ & $\begin{array}{c}P \text { value } \\
\text { (conventional vs } \\
\text { direct TAo-TAVI) }\end{array}$ \\
\hline \multicolumn{5}{|l|}{ Procedural characteristics } \\
\hline Distance to annulus from puncture site $[\mathrm{cm}]$ & $6.3 \pm 1.4$ & $6.2 \pm 1.1$ & $6.8 \pm 2.1$ & .074 \\
\hline Access point & & & & .086 \\
\hline Ministernotomy & $288 / 300(96.0)$ & $216 / 222(97.3)$ & $72 / 78(92.3)$ & \\
\hline Right anterior thoracotomy & $12 / 300(4.0)$ & $6 / 222(2.7)$ & $6 / 78(7.7)$ & \\
\hline Predilation balloon size $[\mathrm{mm}]$ & $20.9 \pm 1.9$ & $20.9 \pm 1.9$ & NA & NA \\
\hline THV size & & & & .728 \\
\hline $23 \mathrm{~mm}$ & $83 / 300(27.7)$ & $59 / 222(26.6)$ & 24/78 (30.8) & \\
\hline $26 \mathrm{~mm}$ & $145 / 300(48.3)$ & $110 / 222(49.6)$ & $35 / 78(44.9)$ & \\
\hline $29 \mathrm{~mm}$ & $72 / 300(24.0)$ & $53 / 222(23.9)$ & $19 / 78(24.4)$ & \\
\hline Procedural duration [min] & $107.0 \pm 37.7$ & $105.6 \pm 37.3$ & $110.9 \pm 38.6$ & .279 \\
\hline Contrast agent volume $[\mathrm{mL}]$ & $101.4 \pm 48.6$ & $104.1 \pm 43.8$ & $94.7 \pm 58.7$ & .157 \\
\hline Balloon postdilation & $70 / 300(23.3)$ & $50 / 222(22.5)$ & 20/78 (25.6) & .575 \\
\hline Periprocedural complications & $28 / 300(9.3)$ & $25 / 222(11.3)$ & $3 / 78(3.9)$ & .053 \\
\hline Access complications* & $6 / 300(2.0)$ & $6 / 222(2.7)$ & $0 / 78(0.0)$ & .345 \\
\hline Device malfunction $\dagger$ & $2 / 300(0.7)$ & $2 / 222(0.9)$ & $0 / 78(0.0)$ & .400 \\
\hline Second valve & $5 / 300(1.7)$ & $4 / 222(1.8)$ & $1 / 78(1.3)$ & .758 \\
\hline Conversion to open surgery & $5 / 300(1.7)$ & $5 / 222(2.3)$ & $0 / 78(0.0)$ & .393 \\
\hline Complete AV block & $15 / 300(5.0)$ & $13 / 222(5.9)$ & $2 / 78(2.6)$ & .251 \\
\hline During BAV predilation & $2 / 15(13.3)$ & $2 / 13(15.4)$ & NA & NA \\
\hline During valve implantation & $6 / 15(40.0)$ & $6 / 13(46.2)$ & $0 / 2(0.0)$ & .215 \\
\hline Complete AV block requiring PPI & $11 / 300(3.7)$ & $11 / 222(5.0)$ & $0 / 78(0.0)$ & .034 \\
\hline \multicolumn{5}{|l|}{ Procedural outcomes } \\
\hline Device success $\underset{\dagger}{\dagger}$ & $296 / 300(98.7)$ & $219 / 222(98.6)$ & 77/78 (98.7) & $>.999$ \\
\hline Periprocedural mortality & $0 / 300(0.0)$ & $0 / 222(0.0)$ & $0 / 78(0.0)$ & NA \\
\hline Moderate/severe paravalvular regurgitation & $10 / 300(3.3)$ & $9 / 222(4.1)$ & $1 / 78(1.3)$ & .463 \\
\hline Peak transvalvular gradient $[\mathrm{mm} \mathrm{Hg}]$ & $17.6 \pm 7.8$ & $17.9 \pm 7.9$ & $16.5 \pm 6.9$ & .328 \\
\hline Mean transvalvular gradient $[\mathrm{mm} \mathrm{Hg}]$ & $9.8 \pm 4.6$ & $9.8 \pm 4.5$ & $9.7 \pm 5.0$ & .857 \\
\hline \multicolumn{5}{|l|}{ Hospitalization } \\
\hline Length of stay in ICU $[d] \S$ & $1.0(1.0-3.0)$ & $1(1.0-2.0)$ & $2.0(1.0-3.0)$ & .052 \\
\hline Time to discharge post-TAVI [d] $\|$ & $8(6.0-11.0)$ & $7(5.8-10.0)$ & $8(7.0-14.0)$ & .005 \\
\hline
\end{tabular}

$S D$, Standard deviation; TAo-TAVI, transaortic transcatheter aortic valve implantation; $N A$, not applicable; $T H V$, transcatheter heart valve; $A V$, atrioventricular; $B A V$, balloon aortic valvuloplasty; $P P I$, permanent pacemaker implantation; $I C U$, intensive care unit; TAVI, transcatheter aortic valve implantation. *Defined as annular/aortic dissection/rupture or uncontrolled bleeding. $\dagger$ Defined as failure to meet any device performance specifications or perform as intended. $\ddagger$ Defined as absence of procedural mortality, correct positioning, and intended performance of THV (VARC-2 criteria (11)). §Data for 9 patients missing (conventional 4, direct 5). ||Data for 3 patients missing (conventional 0, direct 3).

statistically different between groups, and no periprocedural death occurred in either group.

\section{Procedural Efficacy}

Immediately after TAo-TAVI, overall peak and mean transvalvular gradients were reduced to 17.6 and $9.8 \mathrm{~mm}$ $\mathrm{Hg}$, respectively, with no notable differences between conventional and direct groups $(P=.328$ and $P=.857$, respectively) (Table 2). The majority of patients in both groups displayed no/trace PVL after TAVI $(82.1 \%$ and $76.6 \%$ of the direct and conventional groups, respectively; $P=.328$ ) (Figure 2). Likewise, no significant differences were found for any other procedural outcomes.

\section{Thirty-Day Outcomes}

At 30 days postprocedure, 293 patients $(97.7 \%)$ were available for follow-up: $219(98.6 \%)$ in the conventional group and 74 (94.9\%) in the direct group (Table 3). Missing patients were excluded from analyses.

The VARC-2 early safety composite end point was met by comparable proportions of patients in the conventional and direct groups (17.4\% and $22.7 \%$, respectively; $P=.308$ ), with $18.7 \%$ and $20.5 \%$ meeting the clinical efficacy composite end point, respectively $(P=.731)$. After adjusting for baseline characteristics, multivariate analysis indicated that the omission of BAV predilation had no significant effect on the odds of meeting either composite end point at 30 days (early safety: OR, 1.17; 97.5\% CI, 0.53-2.62 and clinical efficacy: OR, 1.14; 95\% CI, 0.51-2.55).

Because there were too few outcomes for the model to function for the individual events or complications over the first 30 days after the TAo-TAVI procedure, we omitted calculating the OR and displayed the descriptive rates only. 


\section{Adult: Aortic Valve}

- Conventiomal TAo-TAVI Direct TAo-TAVI

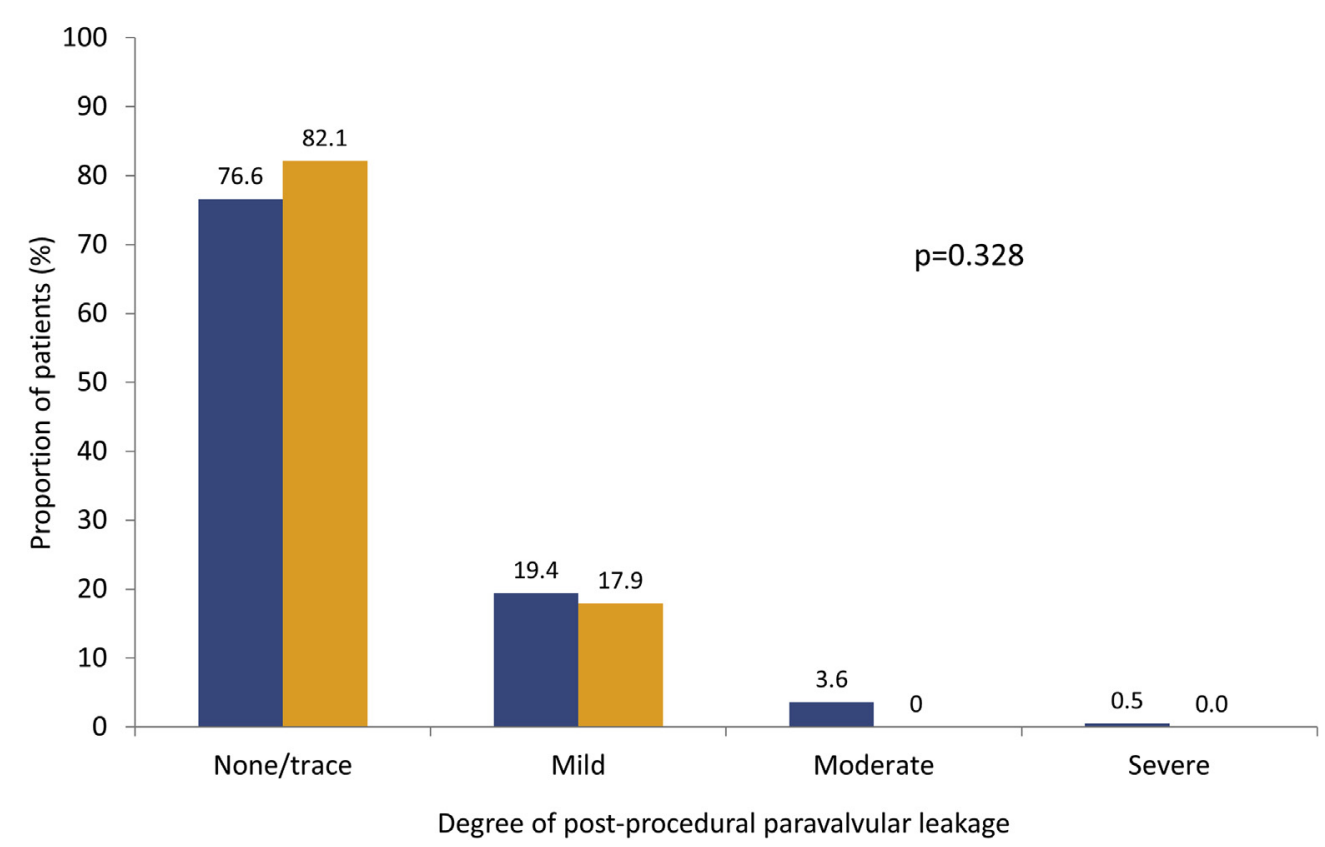

FIGURE 2. Severity of PVL after conventional versus direct TAo-TAVI. TAo-TAVI, Transaortic transcatheter aortic valve implantation; $P V L$, paravalvular leak.

\section{DISCUSSION}

Overall, omission of the BAV predilation step during TAo-TAVI with the balloon-expandable Edwards SAPIEN XT or 3 THV was found to have no significant effect on safety and efficacy outcomes at 30 days, including VARC-2 composite end points. However, the direct approach did appear to reduce the frequency of periprocedural AV block requiring PPI.

\section{Patient Characteristics}

The overall low frequency of PAD in the present patients is surprising, given that this is a common reason for performing TAo-TAVI over TF-TAVI. ${ }^{12}$ However, a recent observational study was in agreement with our finding, reporting only $32 \%$ of TAo-TAVI cases to have PAD. ${ }^{13}$ Other reasons to perform TAVI via the TAo route include femoral artery tortuosity, small access vessel diameter, and surgeon preference, the latter of which is a particular influence in real-world registries.

Few of the baseline characteristics of the 2 groups varied to any great extent. This was with the exception of the presence of PAD, moderate/severe regurgitation insufficiency, and NYHA class, all of which were poorer in the patients who underwent BAV predilation. This indicates that the condition of the native valve was worse in these patients, which may have contributed to the physician's decision to perform BAV. Unfortunately, neither the reason for the physician's choice of conventional or direct TAo-TAVI nor the prevalence of aortic root calcification was recorded in the ROUTE registry; therefore, this cannot be confirmed. However, the degree of valve calcification is known to be less restrictive in TAo-TAVI compared with TF-TAVI because of the shorter access route and increased control. Thus, a proportion of BAV decisions were likely to be based simply on surgeon preference. Finally, a higher proportion of the direct group had previously received BAV, which may be another possible reason for them not requiring it before the TAVI procedure.

\section{Periprocedural Data}

An extremely high rate of device success was achieved in both direct and conventional TAo-TAVI groups. First, this suggests that the decision not to perform BAV predilation was appropriate for the patients in the direct group, with only 1 patient not achieving device success, which was due to a finding of moderate/severe PVL. Recent studies assessing the effects of omitting predilation from TF- and TATAVI procedures have demonstrated the safety and efficacy of this approach. ${ }^{3,14-16}$ Therefore, it is likely that there was a proportion of the conventional group that would have achieved device success in the absence of BAV; however, because of the nonrandomized design of the study, we were unable to confirm this.

The finding that patients undergoing conventional and direct TAo-TAVI had a similar procedural duration and 
TABLE 3. Multivariable analyses showing the effect of omitting balloon aortic valvuloplasty predilation on 30-day outcomes

\begin{tabular}{|c|c|c|c|c|}
\hline & $\begin{array}{c}\text { Total }(\mathbf{N}=293) \\
\mathbf{n}(\%)\end{array}$ & $\begin{array}{c}\text { Conventional } \\
\text { TAo-TAVI }(\mathbf{N}=\mathbf{2 1 9}) \\
\text { n }(\%)\end{array}$ & $\begin{array}{c}\text { Direct TAo-TAVI } \\
(\mathbf{N}=74) \\
\text { n }(\%)\end{array}$ & $\begin{array}{c}\text { Adjusted } \\
\text { OR }(\mathbf{9 7 . 5} \% \mathbf{C I})^{*}\end{array}$ \\
\hline 30-d follow-up & $293 / 300(97.7)$ & 219/222 (98.6) & 74/78 (94.9) & NA \\
\hline \multicolumn{5}{|l|}{ Composite end points } \\
\hline Early safety $\dagger$ & $55 / 294(18.7)$ & $38 / 219(17.4)$ & $17 / 75(22.7)$ & $1.17(0.53-2.62)$ \\
\hline Clinical efficacy $\ddagger$ & $56 / 292(19.2)$ & $41 / 219(18.7)$ & $15 / 73(20.5)$ & $1.14(0.51-2.55)$ \\
\hline All-cause mortality & $18 / 293(6.1)$ & $12 / 219(5.5)$ & $6 / 74(8.1)$ & \\
\hline Nonfatal stroke/TIA & 4/293 (1.4) & $3 / 219(1.4)$ & $1 / 74(1.4)$ & \\
\hline Nonfatal MI & 2/293 (0.7) & $1 / 219(0.5)$ & 1/74 (1.4) & \\
\hline Life-threatening bleeding & $10 / 293(3.4)$ & $8 / 219(3.7)$ & $2 / 74(2.7)$ & \\
\hline AKI (AKIN stage 2 or 3 ) & $27 / 285(9.5)$ & 19/214 (8.9) & 8/71 (11.3) & \\
\hline Major vascular complication & $10 / 293(3.4)$ & $8 / 219(3.7)$ & $2 / 74(2.7)$ & \\
\hline PPI $\S$ & 26/294 (8.8) & 20/220 (9.1) & $6 / 74(8.1)$ & \\
\hline Valve-related dysfunction & $3 / 282(1.1)$ & $3 / 213(1.4)$ & $0 / 69(0)$ & \\
\hline Valve-related symptoms requiring hospitalization & $10 / 282(3.5)$ & $7 / 213(3.3)$ & $3 / 69(4.3)$ & \\
\hline NYHA class III/IV & $34 / 282(12.1)$ & $27 / 213(12.7)$ & $7 / 69(10.1)$ & \\
\hline CCS angina class III/IV & $7 / 241(2.9)$ & $4 / 196(2.0)$ & $3 / 45(6.7)$ & \\
\hline
\end{tabular}

TAo-TAVI, Transaortic transcatheter aortic valve implantation; $O R$, odds ratio; $C I$, confidence interval; NA, not applicable; TIA, transient ischemic attack; $M I$, myocardial infarction; AKI, acute kidney injury; AKIN, Acute Kidney Injury Network; PPI, permanent pacemaker implantation; NYHA, New York Heart Association; CCS, Canadian Cardiovascular Society. *Adjusted for baseline characteristics, age, presence of PAD, moderate/severe regurgitation insufficiency, prior BAV, and NYHA class III or IV. $\dagger$ Composite of all-cause mortality, nonfatal stroke/TIA, life-threatening bleeding, acute kidney injury, major vascular complication, and valve-related dysfunction. $\ddagger$ Composite of all-cause mortality, stroke, hospitalization for valve-related symptoms, NYHA class III or IV, and valve-related dysfunction. §Patients with PPI before being lost to follow-up are included.

volume of contrast agent is unsurprising, given that the short distance between the access site and the aortic annulus means that BAV represents only a small proportion of the overall operative time. A similar result has been reported for direct versus conventional TA-TAVI, ${ }^{17}$ which also involves a short access route. Conversely, direct TF-TAVI has been consistently noted to save time by avoiding lengthy negotiation of the femoral artery with the BAV equipment. ${ }^{3,4,6,7,16}$

It may be expected that, in the absence of BAV predilation, there would be a greater need for postprocedural dilation in the direct TAVI group. However, no significant difference in the rate of balloon postdilation was seen between the 2 groups in the present study. This is consistent with findings from studies assessing the value of BAV predilation in TA- and TF-TAVI procedures using balloonexpandable prostheses ${ }^{3,7,15-17}$ and implies that satisfactory expansion of the THV may not be reliant on the predilation step. This is an important finding, because postdilation has been identified as an independent predictor of major adverse cardiac and cerebrovascular events at 1 year. ${ }^{18}$ However, it should be noted that the baseline characteristics of the patients included in the present analysis indicate that the native valves of those who received $\mathrm{BAV}$ predilation were in a worse condition before TAVI. Therefore, it is possible that in the absence of BAV, more of these patients would have required postdilation.

\section{Periprocedural Complications}

Although no difference in periprocedural mortality was observed between the 2 study groups, there was a trend toward a reduced rate of complications after direct TAoTAVI. The borderline significance for this effect may have been due to the generally low incidence of complications coupled with relatively small patient numbers. This suggests that omitting BAV predilation may hold promise for improving procedural safety for many patients, and this merits investigation in larger studies.

More specifically, a significant difference between groups was seen in relation to the requirement for PPI after complete AV block, which applied to $5 \%$ of conventional patients but to none of the direct patients. It is notable that 2 of 13 instances of complete AV block in the conventional group occurred during the BAV predilation step. A trend toward reduced PPI with direct TAVI has been reported in a number of studies. ${ }^{6,8}$ Furthermore, BAV predilation has been identified as an independent predictive factor for PPI requirement in TAVI (OR, 1.75; 95\% CI, 1.02-3.02; $P=.04) .{ }^{19}$ Prior conduction abnormalities also have been associated with a greater requirement for PPI post-TAVI ${ }^{19}$; however, neither the prevalence of AV block nor right or left bundle branch block varied between the 2 groups at baseline in the present analysis. On the other hand, the potentially inferior condition of the 
native valves in the conventional group, which was indicated by the baseline characteristics, may have been a contributing factor. Data regarding aortic root calcification may help to clarify this hypothesis. In combination with studies investigating direct TF- or TA-TAVI, our data suggest that eliminating BAV from the procedure may reduce the periprocedural need for PPI for some patients.

\section{Early Safety/Efficacy}

No differences in the rates of patients meeting early safety/efficacy end points at 30 days, including the VARC-2 composite measures and each of their elements considered individually, were seen between groups. ${ }^{11}$ This suggests that BAV predilation in TAo-TAVI has little to no short-term clinical value for many patients. Previous studies evaluating the role of predilation in TF- and TATAVI also have come to this conclusion, $3,7,8,14-18$ indicating that this finding appears to be applicable regardless of access route.

Several TF-TAVI studies have associated the omission of BAV predilation with reduced stroke rates ${ }^{16,18}$; however, this was not the case in the present study, with identical rates of nonfatal stroke/transient ischemic attack in the 2 groups. This may be explained by the additional stroke risk posed by the TF access route (due to the abrasion of lengthy arterial lumens and potential dislodgement of atherosclerotic material), and therefore the greater capacity for reducing this risk by omitting the passage of the BAV device. Such an effect likely would be negligible with TAo access because of the short distance between the point of catheter insertion and the aortic valve. In this case, debris from the valve itself would be the cause of stroke. It is possible that a higher rate of stroke would be found in the conventional group compared with the direct group if a larger population was evaluated, with only 4 patients overall experiencing such an event in the present study. Future studies should carefully monitor this important safety outcome.

\section{Study Limitations}

First, the assignment of patients in the conventional or direct groups was dependent on the treatment decision taken by the intervening physician, rather than randomization. Baseline characteristics such as frequency of atherosclerotic disease, moderate/severe aortic regurgitation insufficiency, and NYHA class III/IV, as well as the degree of aortic root calcification, which was not recorded in the registry, would likely have influenced the decision of whether or not to perform BAV. Therefore, there was an imbalance in the characteristics of the 2 groups that would have influenced the observed differences in outcomes. To minimize such effects, 30-day outcomes were adjusted for $\mathrm{PAD}$, moderate/severe regurgitation insufficiency, and NYHA class III/IV at baseline. Second, because evaluating the role of BAV predilation was not an original aim of the ROUTE registry, data relating to justification of its omission/inclusion were not systematically recorded. Accordingly, not all of the variables accounting for selection were necessarily captured, and the multicenter nature of the study means that institutional preferences are likely to have played a role. Third, statistical power was limited by the smaller number of patients who received direct TAoTAVI, with the low event rates potentially resulting in overfitting of the data at multivariable analysis. Because our intention was merely to describe the observed relationship between BAV and outcome variables in the present study, we restricted the multivariable model to clinical safety and efficacy composite end points. Finally, sole use of a balloon-expandable SAPIEN THV means that findings are not necessarily applicable to other valves and deployment systems, such as self-expandable valves.

\section{CONCLUSIONS}

The elimination of BAV predilation from TAo-TAVI with an Edwards SAPIEN balloon-expandable valve appears to be safe and not to affect the success of the procedure in the majority of patients. Although there is likely a proportion of patients who received TAVI who will still require BAV because of the condition of the native valve and aortic root, it appears to be unnecessary in many cases. This is in agreement with prior studies concerning TA- and TF-TAVI. The present real-world data suggest that physicians might consider using a direct rather than a conventional approach in TAo-TAVI. However, larger, randomized studies would be informative to confirm present findings.

\section{Conflict of Interest Statement}

V.B., M.A., H.B., N.B., S.C., R.C., W.E., D.F., G.G., D.J., M.K., J.L., M.L., D.M., K.A.R., M.T., J.-P.V., and M.R. state that they have received research funding and/or speaker honoraria and/or have proctored for Edwards Lifesciences. P.B. states that he has received consultancy fees and that his institution received research grants from Edwards Lifesciences, including one for the conduction of the ROUTE registry. C.D. has no conflict to disclose. M.T. states that he holds stocks in and is employed by Edwards Lifesciences. L.L. has nothing to disclose with regard to commercial support.

Data were captured using the s4trials Software provided by Software for Trials Europe GmbH, Berlin, Germany. Helen Sims (Institute for Pharmacology and Preventive Medicine, Terrassa, Spain) provided editorial support.

\section{References}

1. Patsalis PC, Al-Rashid F, Neumann T, Plicht B, Hildebrandt HA, Wendt D, et al Preparatory balloon aortic valvuloplasty during transcatheter aortic valve implantation for improved valve sizing. JACC Cardiovasc Interv. 2013;6:965-71. 
2. Erdoes G, Basciani R, Huber C, Stortecky S, Wenaweser P, Windecker S, et al. Transcranial Doppler-detected cerebral embolic load during transcatheter aortic valve implantation. Eur J Cardiothorac Surg. 2012;41:778-84.

3. Conradi L, Schaefer A, Seiffert M, Schirmer J, Schaefer U, Schon G, et al. Transfemoral TAVI without pre-dilatation using balloon-expandable devices: a casematched analysis. Clin Res Cardiol. 2015;104:735-42.

4. Kim WK, Praz F, Blumenstein J, Liebetrau C, Gaede L, Van Linden A, et al. Transfemoral aortic valve implantation of Edwards SAPIEN 3 without predilatation. Catheter Cardiovasc Interv. 2017;89:E38-43.

5. Bagur R, Kwok CS, Nombela-Franco L, Ludman PF, de Belder MA, Sponga S, et al. Transcatheter aortic valve implantation with or without preimplantation balloon aortic valvuloplasty: a systematic review and meta-analysis. J Am Heart Assoc. 2016;5:https://doi.org/10.1161/JAHA.115.003191.

6. Islas F, Almeria C, Garcia-Fernandez E, Jimenez P, Nombela-Franco L, Olmos C, et al. Usefulness of echocardiographic criteria for transcatheter aortic valve implantation without balloon predilation: a single-center experience. J Am Soc Echocardiogr. 2015;28:423-9.

7. Mollmann H, Kim WK, Kempfert J, Blumenstein J, Liebetrau C, Nef H, et al. Transfemoral aortic valve implantation of Edwards SAPIEN XT without predilatation is feasible. Clin Cardiol. 2014;37:667-71.

8. Fiorina C, Maffeo D, Curello S, Lipartiti F, Chizzola G, D’Aloia A, et al. Direct transcatheter aortic valve implantation with self-expandable bioprosthesis: feasibility and safety. Cardiovasc Revasc Med. 2014;15:200-3.

9. Viigimaa M, Erglis A, Latkovskis G, Maeots E, Petrulioniene Z, Slapikas R, et al. Prevalence of dyslipidemia in statin-treated patients in the Baltic states (Estonia, Latvia, and Lithuania): Results of the Dyslipidemia International Study (DYSIS). Medicina (Kaunas). 2014;50:44-53.

10. Bapat V, Frank D, Cocchieri R, Jagielak D, Bonaros N, Aiello M, et al. Transcatheter aortic valve replacement using transaortic access: experience from the multicenter, multinational, prospective ROUTE Registry. JACC Cardiovasc Interv. 2016;9:1815-22.

11. Kappetein AP, Head SJ, Genereux P, Piazza N, van Mieghem NM, Blackstone EH, et al. Updated standardized endpoint definitions for transcatheter aortic valve implantation: the Valve Academic Research Consortium-2 consensus document. J Thorac Cardiovasc Surg. 2013;145:6-23.
12. Latsios G, Gerckens U, Grube E. Transaortic transcatheter aortic valve implantation: a novel approach for the truly "no-access option" patients. Catheter Cardiovasc Interv. 2010;75:1129-36.

13. Arai T, Romano M, Lefèvre T, Hovasse T, Farge A, Le Houerou D, et al. Direct comparison of feasibility and safety of transfemoral versus transaortic versus transapical transcatheter aortic valve replacement. JACC Cardiovasc Interv. 2016;9:2320-5.

14. Ferrera C, Nombela-Franco L, Garcia E, Jimenez-Quevedo P, Biagioni C, Gonzalo N, et al. Clinical and hemodynamic results after direct transcatheter aortic valve replacement versus pre-implantation balloon aortic valvuloplasty: a case-matched analysis. Catheter Cardiovasc Interv. 2017;90:809-16.

15. Bandali A, Parry-Williams G, Kassam A, Palmer S, Williams PD, de Belder MA, et al. Direct transfemoral transcatheter aortic valve implantation without balloon pre-dilatation using the Edwards Sapien XT valve. Catheter Cardiovasc Interv. 2016;88:978-85.

16. Aggarwal SK, Delahunty N, Wong B, Tamimi AN, Reinthaler M, Cheang MH, et al. Balloon-expandable transcatheter aortic valves can be successfully and safely implanted transfemorally without balloon valvuloplasty. J Interv Cardiol. 2016;29:319-24.

17. Conradi L, Seiffert M, Schirmer J, Koschyk D, Blankenberg S, Reichenspurner H, et al. Transapical transcatheter aortic valve implantation without prior balloon aortic valvuloplasty: feasible and safe. Eur J Cardiothorac Surg. 2014;46:61-6.

18. Pagnesi M, Jabbour RJ, Latib A, Kawamoto H, Tanaka A, Regazzoli D, et al Usefulness of predilation before transcatheter aortic valve implantation. Am J Cardiol. 2016;118:107-12.

19. Gensas CS, Caixeta A, Siqueira D, Carvalho LA, Sarmento-Leite R, Mangione JA, et al. Predictors of permanent pacemaker requirement after transcatheter aortic valve implantation: insights from a Brazilian registry. Int $J$ Cardiol. 2014;175:248-52.

Key Words: aortic stenosis, balloon aortic valvuloplasty, predilation, transaortic, transcatheter aortic valve implantation 